Supporting Information Available: Tables of positional and displacement parameters, crystallographic data, and bond lengths and angles for compounds 8a and 9.

\title{
Allyl Isomerization Mediated by Cyclopentadienyl Group 6 Metal Derivatives
}

Eva Royo, * Sergio Acebrón, Marta Elena González Mosquera and Pascual Royo*

Departamento de Química Inorgánica, Facultad de Ciencias, Universidad de Alcalá, 28871 Alcalá de Henares, Madrid, Spain 
Molecular structure of $8 \mathrm{a}$ in the solid state

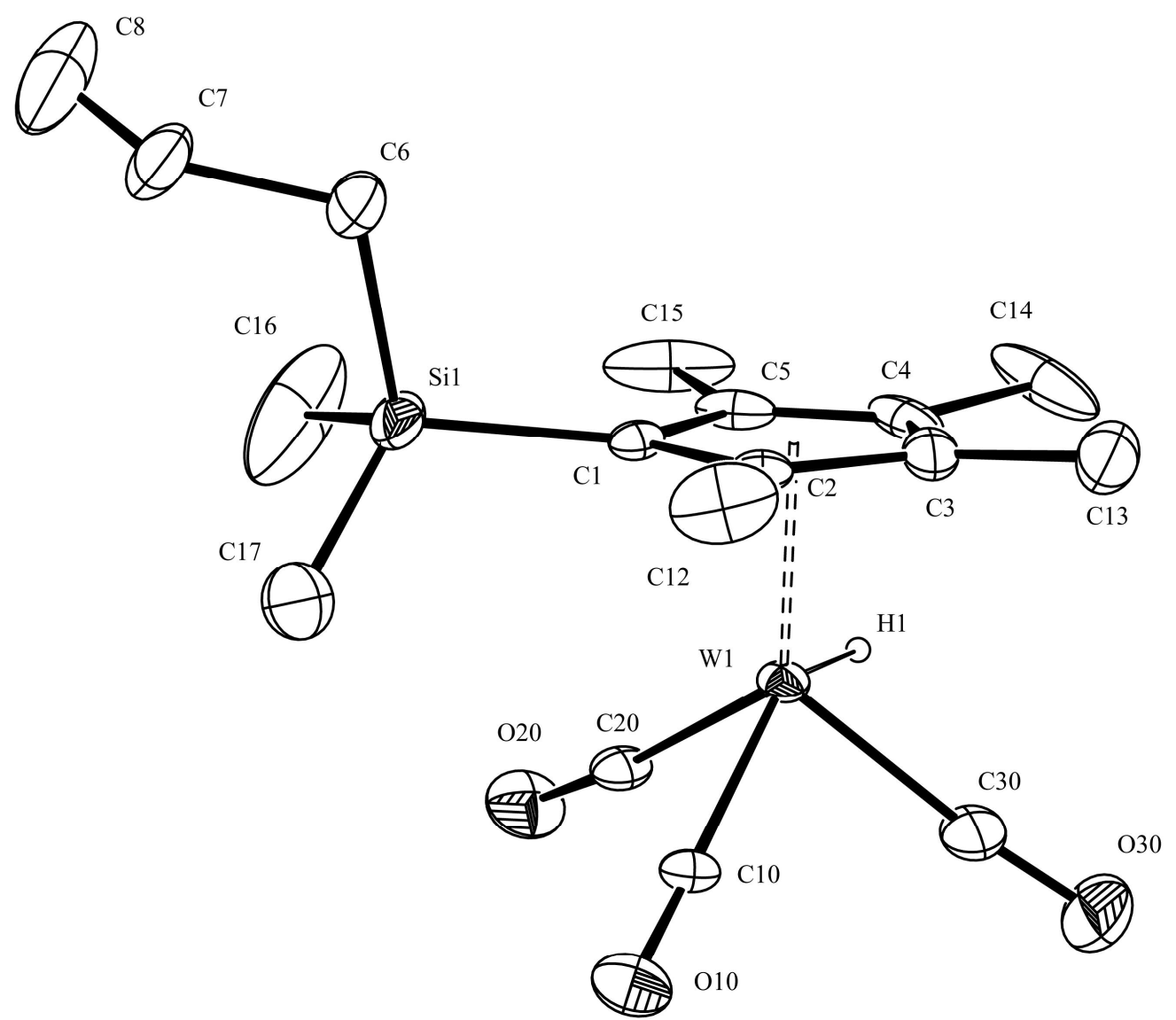

Table 1. Crystal data and structure refinement for 8 a.
Empirical formula
C17 H24 O3 Si W
Formula weight
488.30
Temperature
$200(2) \quad K$
Wavelength
$0.71073 \mathrm{~A}$
Crystal system, space group
Monoclinic, P21/n
Unit cell dimensions
$\mathrm{a}=7.1721(9) \mathrm{A} \quad$ alpha $=90 \mathrm{deg}$.
$\mathrm{b}=17.954(4) \mathrm{A}$ beta $=96.236(13) \mathrm{deg}$.
$\mathrm{c}=15.3349(19) \mathrm{A}$ gamma $=90 \mathrm{deg}$.
Volume
$1963.0(6) \quad A \wedge 3$
Z, Calculated density
4, $1.652 \mathrm{Mg} / \mathrm{m} \wedge 3$
Absorption coefficient
$5.954 m m^{\wedge}-1$ 


\begin{tabular}{|c|c|}
\hline$F(000)$ & 952 \\
\hline Crystal size & $0.38 \times 0.28 \times 0.25 \mathrm{~mm}$ \\
\hline Theta range for data collection & 3.65 to 27.50 deg. \\
\hline Limiting indices & $-9<=\mathrm{h}<=9, \quad-23<=\mathrm{k}<=23, \quad-19<=1<=19$ \\
\hline Reflections collected / unique & $42907 / 4470 \quad[\mathrm{R}($ int $)=0.0609]$ \\
\hline Completeness to theta $=27.50$ & $99.2 \%$ \\
\hline Max. and min. transmission & 1.433 and 0.728 \\
\hline Refinement method & Full-matrix least-squares on $F^{\wedge} 2$ \\
\hline Data / restraints / parameters & $4470 / 0 / 199$ \\
\hline Goodness-of-fit on $F^{\wedge} 2$ & 1.080 \\
\hline Final R indices [I>2sigma(I)] & $\mathrm{R} 1=0.0465, \mathrm{wR} 2=0.1029$ \\
\hline R indices (all data) & $\mathrm{R} 1=0.0682, \mathrm{wR} 2=0.1124$ \\
\hline Largest diff. peak and & 1.679 and -1.8350 e.A^-3 \\
\hline
\end{tabular}

Table 2. Atomic coordinates $(\mathrm{x} 10 \wedge 4)$ and equivalent isotropic displacement parameters $(A \wedge 2 \times 10 \wedge 3)$ for $8 \mathrm{a}$. U(eq) is defined as one third of the trace of the orthogonalized Uij tensor.

\begin{tabular}{|c|c|c|c|c|}
\hline & $\mathrm{x}$ & $\mathrm{y}$ & $\mathrm{z}$ & $\mathrm{U}(\mathrm{eq})$ \\
\hline $\mathrm{W}(1)$ & $9568(1)$ & $2125(1)$ & $4523(1)$ & $37(1)$ \\
\hline $\mathrm{Si}(1)$ & $7077(4)$ & $3831(2)$ & $3550(3)$ & $77(1)$ \\
\hline$C(1)$ & $7008(10)$ & $2867(4)$ & $4033(5)$ & $37(2)$ \\
\hline $\mathrm{C}(2)$ & $6849(9)$ & $2185(4)$ & $3508(5)$ & $37(2)$ \\
\hline$C(3)$ & $6571(11)$ & $1578(5)$ & $4080(7)$ & $56(2)$ \\
\hline$C(4)$ & $6575(11)$ & $1854(7)$ & $4949(6)$ & $64(3)$ \\
\hline$C(5)$ & $6822(11)$ & $2633(7)$ & $4920(5)$ & $55(2)$ \\
\hline $\mathrm{C}(12)$ & $6821(18)$ & $2123(8)$ & $2528(6)$ & $85(4)$ \\
\hline$C(13)$ & $6168(17)$ & $790(6)$ & $3806(12)$ & $114(6)$ \\
\hline $\mathrm{C}(14)$ & $6080(50)$ & $1530(20)$ & $5790(20)$ & $101(17)$ \\
\hline$C(15)$ & $6860(50)$ & $3281(18)$ & $5649(18)$ & $75(9)$ \\
\hline$C\left(14^{\prime}\right)$ & $6280(70)$ & $1180(30)$ & $5630(40)$ & $86(19)$ \\
\hline$C\left(15^{\prime}\right)$ & $6670(50)$ & $2900(30)$ & $5840(30)$ & $75(14)$ \\
\hline$C(6)$ & $4666(16)$ & $4016(7)$ & $3012(10)$ & $92(4)$ \\
\hline$C(7)$ & $4430(20)$ & $4756(9)$ & $2547(17)$ & $171(11)$ \\
\hline$C(8)$ & $3770(30)$ & $5289(9)$ & $2608(13)$ & $143(8)$ \\
\hline$C(16)$ & $7260(90)$ & $4630(30)$ & $4630(40)$ & $170(20)$ \\
\hline $\mathrm{C}(17)$ & $8990(40)$ & $4047(17)$ & $3040(20)$ & $94(10)$ \\
\hline$C\left(16^{\prime}\right)$ & $7940(90)$ & $4540(18)$ & $4220(30)$ & $170(30)$ \\
\hline$C\left(17^{\prime}\right)$ & $8530(50)$ & $3770(20)$ & $2475(18)$ & $107(12)$ \\
\hline$C(10)$ & $11294(11)$ & $2214(5)$ & $3591(5)$ & $43(2)$ \\
\hline$C(20)$ & $11243(11)$ & $2910(5)$ & $5059(6)$ & $50(2)$ \\
\hline
\end{tabular}




\begin{tabular}{lllll}
$\mathrm{O}(10)$ & $12258(10)$ & $2270(4)$ & $3050(4)$ & $68(2)$ \\
$\mathrm{O}(20)$ & $12113(11)$ & $3414(5)$ & $5384(6)$ & $88(3)$ \\
$\mathrm{C}(30)$ & $10952(14)$ & $1184(7)$ & $4737(9)$ & $63(3)$ \\
$\mathrm{O}(30)$ & $11595(14)$ & $596(5)$ & $4840(8)$ & $99(3)$ \\
$\mathrm{C}\left(30^{\prime}\right)$ & $10830(60)$ & $1450(20)$ & $5420(30)$ & $26(9)$ \\
$\mathrm{O}\left(30^{\prime}\right)$ & $11440(60)$ & $1010(30)$ & $5900(30)$ & $69(14)$ \\
\hline
\end{tabular}

Table 3. Bond lengths [A] and angles [deg] for 8a.

\begin{tabular}{|c|c|}
\hline$W(1)-C(30)$ & $1.977(12)$ \\
\hline$W(1)-C(20)$ & $1.973(9)$ \\
\hline$W(1)-C\left(30^{\prime}\right)$ & $1.95(4)$ \\
\hline$W(1)-C(10)$ & $1.996(8)$ \\
\hline$W(1)-C(5)$ & $2.311(8)$ \\
\hline $\mathrm{W}(1)-\mathrm{C}(1)$ & $2.326(7)$ \\
\hline $\mathrm{W}(1)-\mathrm{C}(2)$ & $2.362(6)$ \\
\hline $\mathrm{W}(1)-\mathrm{C}(4)$ & $2.362(8)$ \\
\hline$W(1)-C(3)$ & $2.394(8)$ \\
\hline $\mathrm{W}(1)-\mathrm{H}(1)$ & $1.7014(4)$ \\
\hline $\mathrm{Si}(1)-\mathrm{C}(17)$ & $1.69(3)$ \\
\hline $\mathrm{Si}(1)-\mathrm{C}\left(16^{\prime}\right)$ & $1.71(3)$ \\
\hline $\mathrm{Si}(1)-\mathrm{C}(6)$ & $1.862(11)$ \\
\hline $\mathrm{Si}(1)-\mathrm{C}\left(17^{\prime}\right)$ & $2.05(3)$ \\
\hline $\mathrm{Si}(1)-\mathrm{C}(1)$ & $1.887(8)$ \\
\hline $\mathrm{Si}(1)-\mathrm{C}(16)$ & $2.19(3)$ \\
\hline$C(1)-C(5)$ & $1.444(11)$ \\
\hline$C(1)-C(2)$ & $1.464(11)$ \\
\hline$C(2)-C(3)$ & $1.426(12)$ \\
\hline$C(2)-C(12)$ & $1.504(11)$ \\
\hline$C(3)-C(4)$ & $1.423(14)$ \\
\hline$C(3)-C(13)$ & $1.498(15)$ \\
\hline$C(4)-C(5)$ & $1.411(15)$ \\
\hline$C(4)-C(14)$ & $1.50(4)$ \\
\hline$C(4)-C\left(14^{\prime}\right)$ & $1.61(4)$ \\
\hline$C(5)-C\left(15^{\prime}\right)$ & $1.50(4)$ \\
\hline$C(5)-C(15)$ & $1.63(4)$ \\
\hline $\mathrm{C}(12)-\mathrm{H}(12 \mathrm{~A})$ & 0.9800 \\
\hline $\mathrm{C}(12)-\mathrm{H}(12 \mathrm{~B})$ & 0.9800 \\
\hline $\mathrm{C}(12)-\mathrm{H}(12 \mathrm{C})$ & 0.9800 \\
\hline $\mathrm{C}(13)-\mathrm{H}(13 \mathrm{~A})$ & 0.9800 \\
\hline $\mathrm{C}(13)-\mathrm{H}(13 \mathrm{~B})$ & 0.9800 \\
\hline$C(13)-H(13 C)$ & 0.9800 \\
\hline $\mathrm{C}(14)-\mathrm{H}(14 \mathrm{~A})$ & 0.9800 \\
\hline$C(14)-H(14 B)$ & 0.9800 \\
\hline $\mathrm{C}(14)-\mathrm{H}(14 \mathrm{C})$ & 0.9800 \\
\hline $\mathrm{C}(15)-\mathrm{H}(15 \mathrm{~A})$ & 0.9800 \\
\hline$C(15)-H(15 B)$ & 0.9800 \\
\hline $\mathrm{C}(15)-\mathrm{H}(15 \mathrm{C})$ & 0.9800 \\
\hline $\mathrm{C}\left(14^{\prime}\right)-\mathrm{H}(14 \mathrm{D})$ & 0.9800 \\
\hline $\mathrm{C}\left(14^{\prime}\right)-\mathrm{H}(14 \mathrm{E})$ & 0.9800 \\
\hline $\mathrm{C}\left(14^{\prime}\right)-\mathrm{H}(14 \mathrm{~F})$ & 0.9800 \\
\hline $\mathrm{C}\left(15^{\prime}\right)-\mathrm{H}(15 \mathrm{D})$ & 0.9800 \\
\hline $\mathrm{C}\left(15^{\prime}\right)-\mathrm{H}(15 \mathrm{E})$ & 0.9800 \\
\hline $\mathrm{C}\left(15^{\prime}\right)-\mathrm{H}(15 \mathrm{~F})$ & 0.9800 \\
\hline$C(6)-C(7)$ & $1.508(17)$ \\
\hline$C(6)-H(6 A)$ & 0.9900 \\
\hline$C(6)-H(6 B)$ & 0.9900 \\
\hline$C(7)-C(8)$ & $1.07(2)$ \\
\hline
\end{tabular}




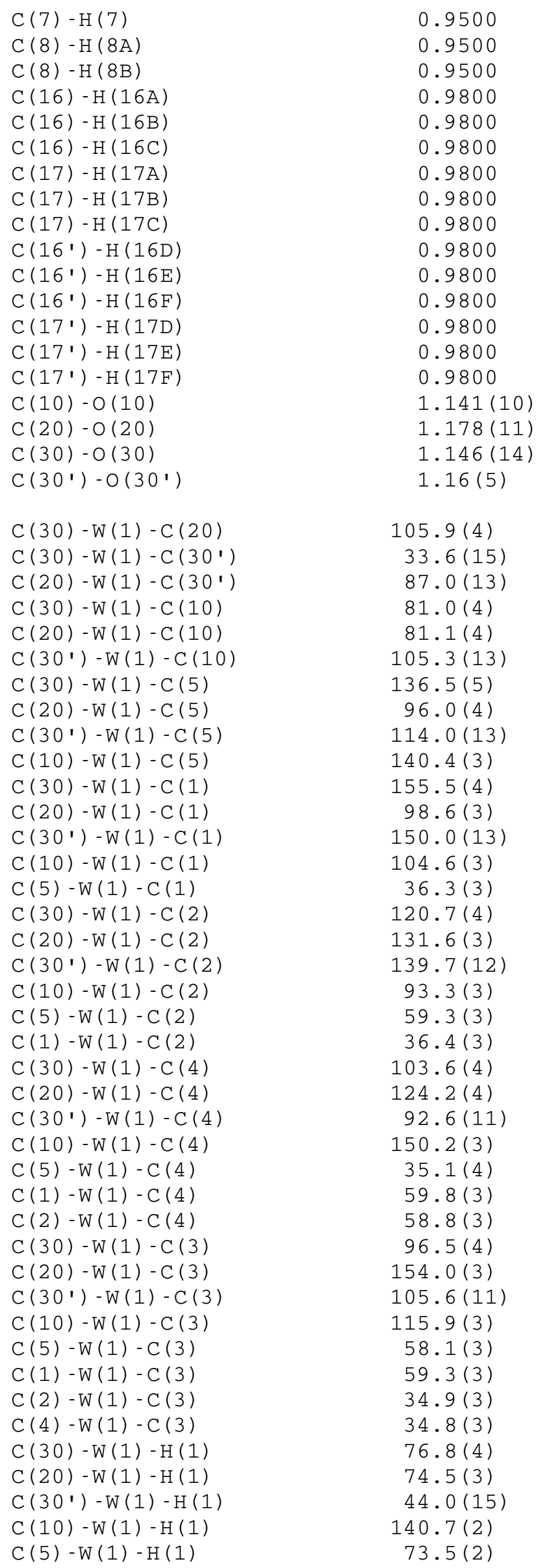




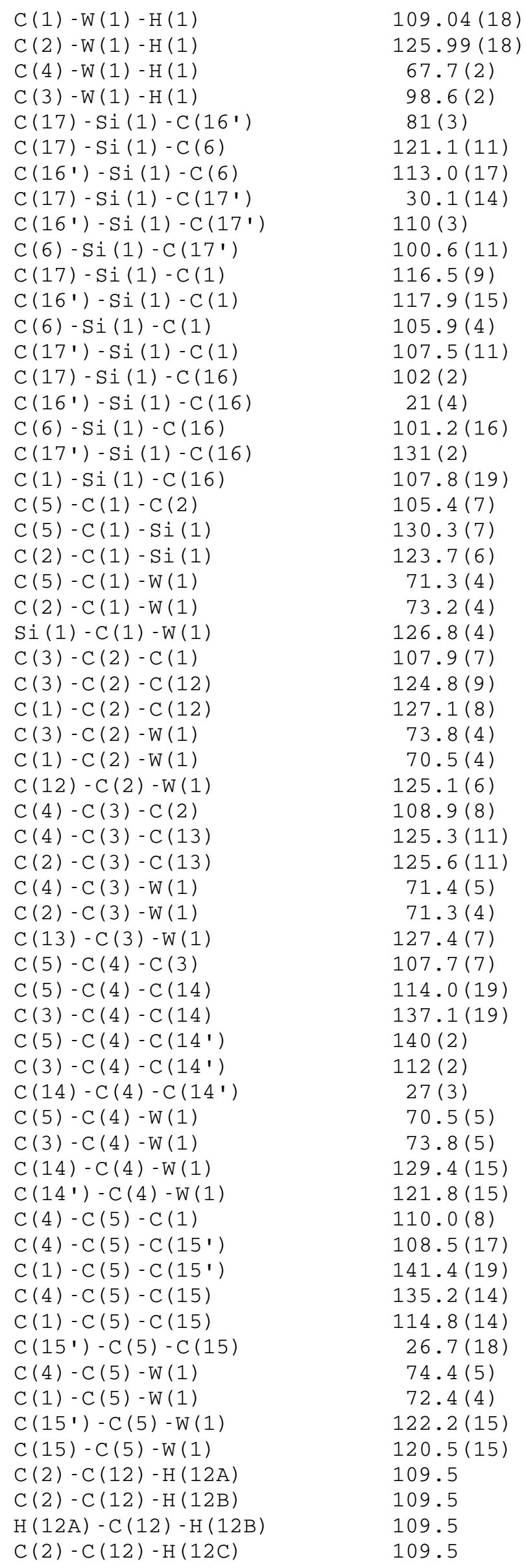




\begin{tabular}{|c|c|}
\hline $\mathrm{H}(12 \mathrm{~A})-\mathrm{C}(12)-\mathrm{H}(12 \mathrm{C})$ & 109.5 \\
\hline $\mathrm{H}(12 \mathrm{~B})-\mathrm{C}(12)-\mathrm{H}(12 \mathrm{C})$ & 109.5 \\
\hline $\mathrm{C}(3)-\mathrm{C}(13)-\mathrm{H}(13 \mathrm{~A})$ & 109.5 \\
\hline$C(3)-C(13)-H(13 B)$ & 109.5 \\
\hline$H(13 A)-C(13)-H(13 B)$ & 109.5 \\
\hline$C(3)-C(13)-H(13 C)$ & 109.5 \\
\hline$H(13 A)-C(13)-H(13 C)$ & 109.5 \\
\hline$H(13 B)-C(13)-H(13 C)$ & 109.5 \\
\hline$C(4)-C(14)-H(14 A)$ & 109.5 \\
\hline$C(4)-C(14)-H(14 B)$ & 109.5 \\
\hline $\mathrm{H}(14 \mathrm{~A})-\mathrm{C}(14)-\mathrm{H}(14 \mathrm{~B})$ & 109.5 \\
\hline $\mathrm{C}(4)-\mathrm{C}(14)-\mathrm{H}(14 \mathrm{C})$ & 109.5 \\
\hline $\mathrm{H}(14 \mathrm{~A})-\mathrm{C}(14)-\mathrm{H}(14 \mathrm{C})$ & 109.5 \\
\hline $\mathrm{H}(14 \mathrm{~B})-\mathrm{C}(14)-\mathrm{H}(14 \mathrm{C})$ & 109.5 \\
\hline$C(5)-C(15)-H(15 A)$ & 109.5 \\
\hline$C(5)-C(15)-H(15 B)$ & 109.5 \\
\hline$H(15 A)-C(15)-H(15 B)$ & 109.5 \\
\hline$C(5)-C(15)-H(15 C)$ & 109.5 \\
\hline $\mathrm{H}(15 \mathrm{~A})-\mathrm{C}(15)-\mathrm{H}(15 \mathrm{C})$ & 109.5 \\
\hline$H(15 B)-C(15)-H(15 C)$ & 109.5 \\
\hline $\mathrm{C}(4)-\mathrm{C}\left(14{ }^{\prime}\right)-\mathrm{H}(14 \mathrm{D})$ & 109.5 \\
\hline$C(4)-C\left(14^{\prime}\right)-H(14 E)$ & 109.5 \\
\hline $\mathrm{H}(14 \mathrm{D})-\mathrm{C}\left(14^{\prime}\right)-\mathrm{H}(14 \mathrm{E})$ & 109.5 \\
\hline $\mathrm{C}(4)-\mathrm{C}\left(14^{\prime}\right)-\mathrm{H}(14 \mathrm{~F})$ & 109.5 \\
\hline $\mathrm{H}(14 \mathrm{D})-\mathrm{C}\left(14^{\prime}\right)-\mathrm{H}(14 \mathrm{~F})$ & 109.5 \\
\hline $\mathrm{H}(14 \mathrm{E})-\mathrm{C}\left(14^{\prime}\right)-\mathrm{H}(14 \mathrm{~F})$ & 109.5 \\
\hline$C(5)-C\left(15^{\prime}\right)-H(15 D)$ & 109.5 \\
\hline$C(5)-C\left(15^{\prime}\right)-H(15 E)$ & 109.5 \\
\hline $\mathrm{H}(15 \mathrm{D})-\mathrm{C}\left(15^{\prime}\right)-\mathrm{H}(15 \mathrm{E})$ & 109.5 \\
\hline$C(5)-C\left(15^{\prime}\right)-H(15 F)$ & 109.5 \\
\hline $\mathrm{H}(15 \mathrm{D})-\mathrm{C}\left(15^{\prime}\right)-\mathrm{H}(15 \mathrm{~F})$ & 109.5 \\
\hline $\mathrm{H}(15 \mathrm{E})-\mathrm{C}\left(15^{\prime}\right)-\mathrm{H}(15 \mathrm{~F})$ & 109.5 \\
\hline$C(7)-C(6)-S i(1)$ & $114.7(9)$ \\
\hline$C(7)-C(6)-H(6 A)$ & 108.6 \\
\hline $\mathrm{Si}(1)-\mathrm{C}(6)-\mathrm{H}(6 \mathrm{~A})$ & 108.6 \\
\hline$C(7)-C(6)-H(6 B)$ & 108.6 \\
\hline $\mathrm{Si}(1)-\mathrm{C}(6)-\mathrm{H}(6 \mathrm{~B})$ & 108.6 \\
\hline$H(6 A)-C(6)-H(6 B)$ & 107.6 \\
\hline$C(8)-C(7)-C(6)$ & $140(3)$ \\
\hline$C(8)-C(7)-H(7)$ & 109.8 \\
\hline$C(6)-C(7)-H(7)$ & 109.8 \\
\hline$C(7)-C(8)-H(8 A)$ & 120.0 \\
\hline$C(7)-C(8)-H(8 B)$ & 120.0 \\
\hline $\mathrm{H}(8 \mathrm{~A})-\mathrm{C}(8)-\mathrm{H}(8 \mathrm{~B})$ & 120.0 \\
\hline $\mathrm{Si}(1)-\mathrm{C}(16)-\mathrm{H}(16 \mathrm{~A})$ & 109.5 \\
\hline $\mathrm{Si}(1)-\mathrm{C}(16)-\mathrm{H}(16 \mathrm{~B})$ & 109.5 \\
\hline$H(16 A)-C(16)-H(16 B)$ & 109.5 \\
\hline $\mathrm{Si}(1)-\mathrm{C}(16)-\mathrm{H}(16 \mathrm{C})$ & 109.5 \\
\hline$H(16 A)-C(16)-H(16 C)$ & 109.5 \\
\hline$H(16 B)-C(16)-H(16 C)$ & 109.5 \\
\hline $\mathrm{Si}(1)-\mathrm{C}(17)-\mathrm{H}(17 \mathrm{~A})$ & 109.5 \\
\hline $\mathrm{Si}(1)-\mathrm{C}(17)-\mathrm{H}(17 \mathrm{~B})$ & 109.5 \\
\hline $\mathrm{H}(17 \mathrm{~A})-\mathrm{C}(17)-\mathrm{H}(17 \mathrm{~B})$ & 109.5 \\
\hline $\mathrm{Si}(1)-\mathrm{C}(17)-\mathrm{H}(17 \mathrm{C})$ & 109.5 \\
\hline $\mathrm{H}(17 \mathrm{~A})-\mathrm{C}(17)-\mathrm{H}(17 \mathrm{C})$ & 109.5 \\
\hline $\mathrm{H}(17 \mathrm{~B})-\mathrm{C}(17)-\mathrm{H}(17 \mathrm{C})$ & 109.5 \\
\hline $\mathrm{Si}(1)-\mathrm{C}\left(16^{\prime}\right)-\mathrm{H}(16 \mathrm{D})$ & 109.5 \\
\hline $\mathrm{Si}(1)-\mathrm{C}\left(16^{\prime}\right)-\mathrm{H}(16 \mathrm{E})$ & 109.5 \\
\hline $\mathrm{H}(16 \mathrm{D})-\mathrm{C}\left(16^{\prime}\right)-\mathrm{H}(16 \mathrm{E})$ & 109.5 \\
\hline $\mathrm{Si}(1)-\mathrm{C}\left(16^{\prime}\right)-\mathrm{H}(16 \mathrm{~F})$ & 109.5 \\
\hline $\mathrm{H}(16 \mathrm{D})-\mathrm{C}\left(16^{\prime}\right)-\mathrm{H}(16 \mathrm{~F})$ & 109.5 \\
\hline
\end{tabular}




\begin{tabular}{|c|c|}
\hline $\mathrm{H}(16 \mathrm{E})-\mathrm{C}\left(16^{\prime}\right)-\mathrm{H}(16 \mathrm{~F})$ & 109.5 \\
\hline $\mathrm{Si}(1)-\mathrm{C}\left(17 \mathbf{I}^{\prime}\right)-\mathrm{H}(17 \mathrm{D})$ & 109.5 \\
\hline $\mathrm{Si}(1)-\mathrm{C}\left(17 \mathbf{\prime}^{\prime}\right)-\mathrm{H}(17 \mathrm{E})$ & 109.5 \\
\hline $\mathrm{H}(17 \mathrm{D})-\mathrm{C}(17 \cdot)-\mathrm{H}(17 \mathrm{E})$ & 109.5 \\
\hline $\mathrm{Si}(1)-\mathrm{C}\left(17^{\prime}\right)-\mathrm{H}(17 \mathrm{~F})$ & 109.5 \\
\hline $\mathrm{H}(17 \mathrm{D})-\mathrm{C}\left(17 \mathbf{\prime}^{\prime}\right)-\mathrm{H}(17 \mathrm{~F})$ & 109.5 \\
\hline $\mathrm{H}(17 \mathrm{E})-\mathrm{C}\left(17{ }^{\prime}\right)-\mathrm{H}(17 \mathrm{~F})$ & 109.5 \\
\hline$O(10)-C(10)-W(1)$ & $178.9(8)$ \\
\hline$O(20)-C(20)-W(1)$ & $174.6(8)$ \\
\hline$O(30)-C(30)-W(1)$ & $173.3(11)$ \\
\hline$O\left(30^{\prime}\right)-C\left(30^{\prime}\right)-W(1)$ & $175(4)$ \\
\hline $\mathrm{O}\left(30^{\prime}\right)-\mathrm{C}\left(30^{\prime}\right)-\mathrm{H}(1)$ & $123(5)$ \\
\hline $\mathrm{W}(1)-\mathrm{C}\left(30^{\prime}\right)-\mathrm{H}(1)$ & $58.4(14)$ \\
\hline
\end{tabular}

Symmetry transformations used to generate equivalent atoms:

Table 4. Anisotropic displacement parameters $(A \wedge 2 \times 10 \wedge 3)$ for $8 a$. The anisotropic displacement factor exponent takes the form: $-2 \mathrm{pi} \wedge 2[\mathrm{~h} \wedge 2 \mathrm{a} * \wedge 2 \mathrm{U} 11+\ldots+2 \mathrm{~h} k \mathrm{k}$ * b* U12]

\begin{tabular}{|c|c|c|c|c|c|c|}
\hline & U11 & U2 2 & U33 & U23 & U13 & U12 \\
\hline $\mathrm{W}(1)$ & $24(1)$ & 49 (1) & $37(1)$ & $9(1)$ & $-3(1)$ & $1(1)$ \\
\hline Si (1) & 41 (1) & $44(2)$ & $140(3)$ & $20(2)$ & $-21(2)$ & $-2(1)$ \\
\hline $\mathrm{C}(1)$ & $23(3)$ & $46(4)$ & $40(4)$ & $-4(3)$ & $-3(3)$ & $5(3)$ \\
\hline$C(2)$ & $21(3)$ & $55(5)$ & $34(3)$ & $-4(3)$ & $-6(3)$ & $1(3)$ \\
\hline$C(3)$ & $31(4)$ & $52(5)$ & $81(6)$ & $15(5)$ & $-12(4)$ & $-4(4)$ \\
\hline$C(4)$ & $25(4)$ & $115(9)$ & $48(5)$ & $40(5)$ & $-4(3)$ & $-6(5)$ \\
\hline$C(5)$ & $25(4)$ & $105(8)$ & $33(4)$ & $-10(4)$ & $2(3)$ & $6(4)$ \\
\hline C (12) & $82(8)$ & $137(11)$ & $36(5)$ & $-25(6)$ & $4(5)$ & $20(7)$ \\
\hline$C(13)$ & $57(7)$ & $50(7)$ & $226(18)$ & $7(9)$ & $-40(9)$ & $-4(5)$ \\
\hline $\mathrm{C}(14)$ & $54(16)$ & $140(40)$ & $63(18)$ & $60(20)$ & $-24(14)$ & $-20(20)$ \\
\hline$C(15)$ & $59(17)$ & $120(30)$ & $32(12)$ & $-29(15)$ & $7(10)$ & $-10(17)$ \\
\hline $\mathrm{C}\left(14^{\prime}\right)$ & $46(14)$ & $110(30)$ & $100(30)$ & $70(20)$ & $14(14)$ & $-25(18)$ \\
\hline$C\left(15^{\prime}\right)$ & $28(11)$ & $160(40)$ & $70(20)$ & $-50(20)$ & $13(11)$ & $0(20)$ \\
\hline$C(6)$ & $58(7)$ & $63(7)$ & $149(12)$ & $42(7)$ & $-19(7)$ & $0(5)$ \\
\hline$C(7)$ & $73(9)$ & $69(10)$ & $350(30)$ & $87(14)$ & $-44(13)$ & $6(7)$ \\
\hline$C(8)$ & $175(19)$ & $73(10)$ & $164(17)$ & $22(10)$ & $-57(14)$ & $24(11)$ \\
\hline$C(16)$ & $140(30)$ & $110(30)$ & $240(60)$ & $-130(30)$ & $-40(30)$ & $10(30)$ \\
\hline C (17) & $65(16)$ & $80(20)$ & $130(30)$ & $70(20)$ & $21(17)$ & $4(13)$ \\
\hline$C\left(16^{\prime}\right)$ & $280(70)$ & $29(14)$ & $180(40)$ & $-50(20)$ & $-150(50)$ & $40(20)$ \\
\hline C (17') & $110(30)$ & $140(30)$ & $73(18)$ & $30(19)$ & $9(17)$ & $10(20)$ \\
\hline $\mathrm{C}(10)$ & $27(4)$ & $52(5)$ & $49(4)$ & $-2(4)$ & $4(3)$ & $2(3)$ \\
\hline$C(20)$ & $28(4)$ & $64(6)$ & $56(5)$ & $-6(4)$ & $-6(3)$ & $3(4)$ \\
\hline$O(10)$ & $56(4)$ & $94(5)$ & $57(4)$ & $-5(4)$ & 19 (3) & $-4(4)$ \\
\hline$O(20)$ & $66(5)$ & $100(6)$ & $93(6)$ & $-27(5)$ & $-15(4)$ & $-21(5)$ \\
\hline$C(30)$ & $30(5)$ & $69(9)$ & $69(8)$ & $14(7)$ & $-3(5)$ & $8(5)$ \\
\hline$O(30)$ & $64(6)$ & $57(6)$ & $146(10)$ & $27(6)$ & $-8(6)$ & $18(5)$ \\
\hline$C\left(30^{\prime}\right)$ & $29(19)$ & $30(20)$ & $80(30)$ & $20(20)$ & $10(20)$ & $-6(15)$ \\
\hline$O\left(30^{\prime}\right)$ & $100(30)$ & $120(40)$ & $80(30)$ & $40(30)$ & $-60(20)$ & $0(30)$ \\
\hline
\end{tabular}




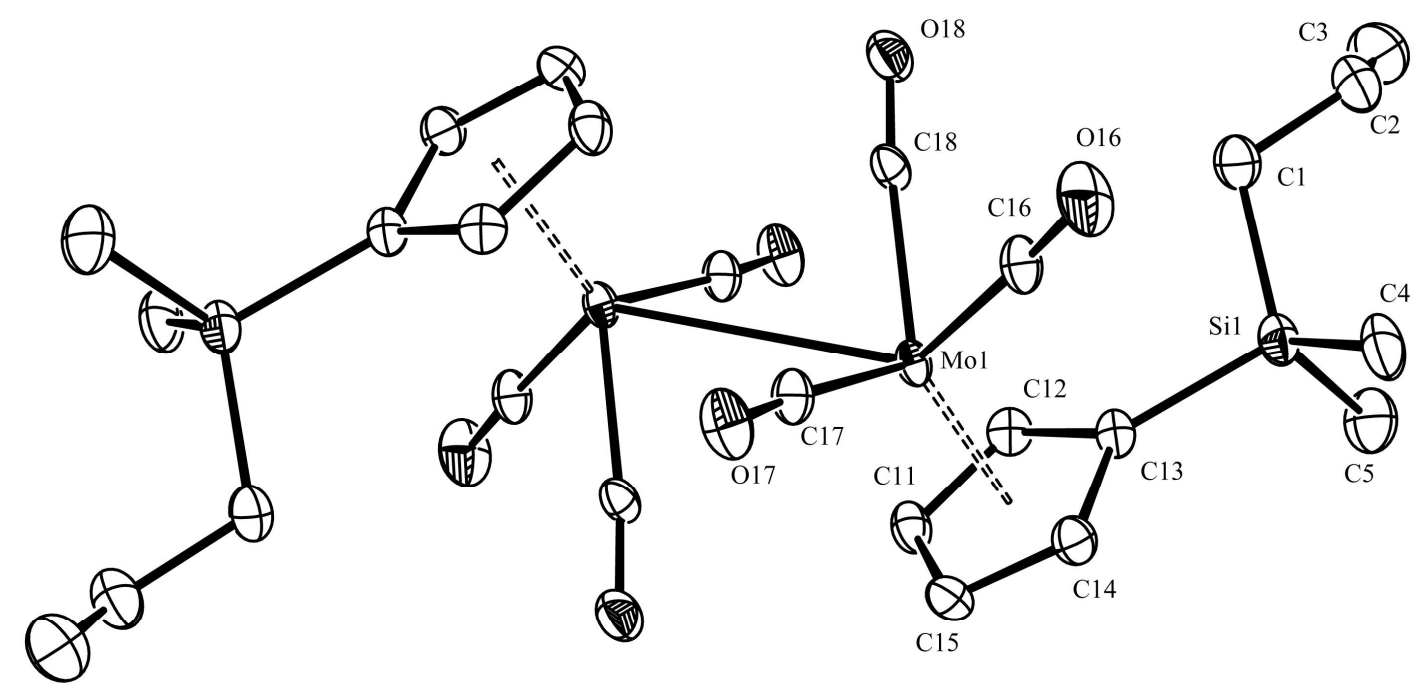

Table 1. Crystal data and structure refinement for 9.

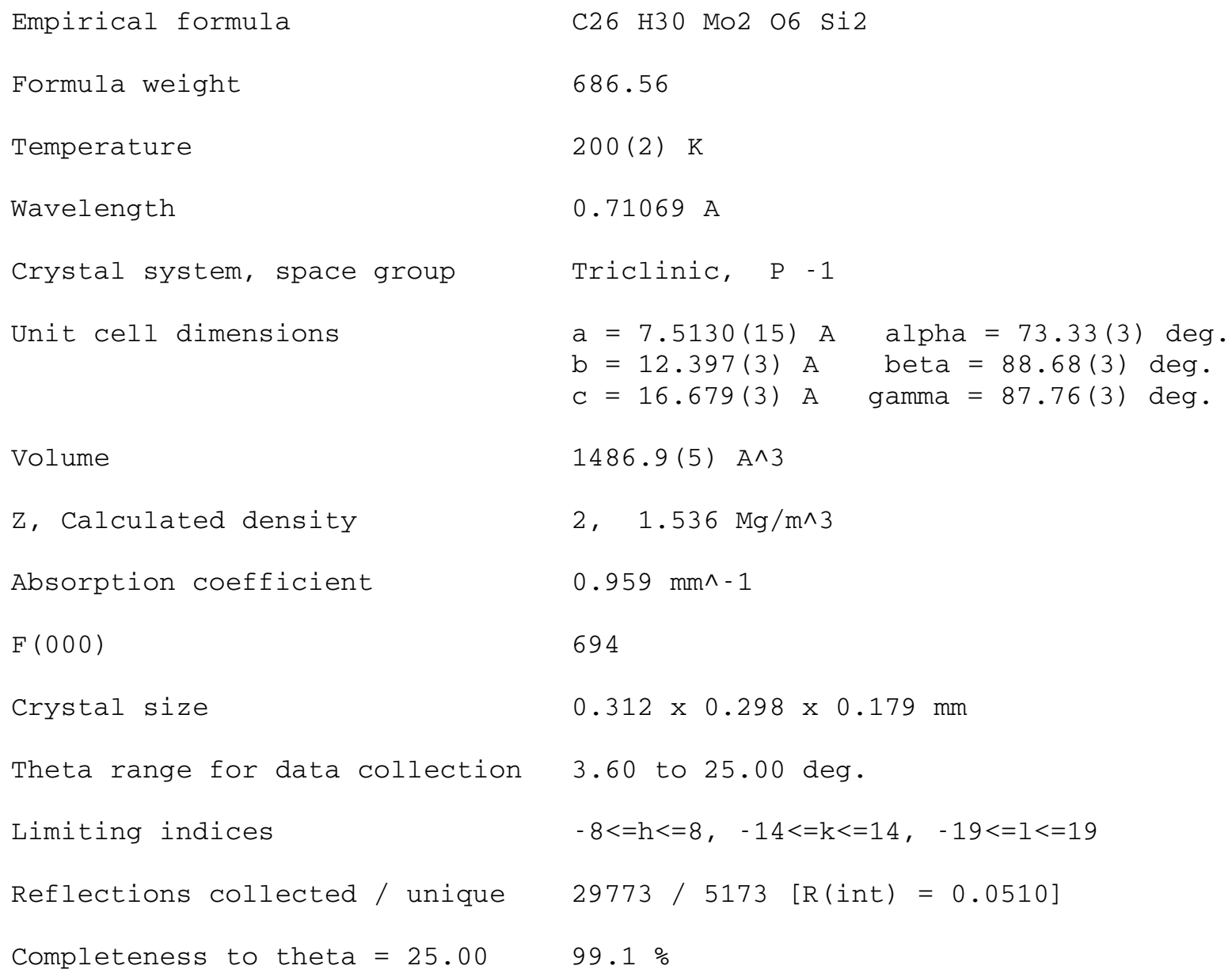


Absorption correction

Max. and min. transmission

Refinement method

Data / restraints / parameters

Goodness-of-fit on $F \wedge 2$

Final $R$ indices [I $>2$ sigma(I)]

$\mathrm{R}$ indices (all data)

Largest diff. peak and hole
Semi-empirical from equivalents

1.175 and 0.886

Full-matrix least-squares on $\mathrm{F}^{\wedge} 2$

$5173 / 0 / 325$

0.995

$\mathrm{R} 1=0.0528, \mathrm{wR} 2=0.1304$

$\mathrm{R} 1=0.0735, \mathrm{wR} 2=0.1391$

2.738 and -0.881 e.A^-3

Table 2. Atomic coordinates $(\mathrm{x} 10 \wedge 4)$ and equivalent isotropic displacement parameters $(A \wedge 2 \times 10 \wedge 3)$ for 9.

$\mathrm{U}(\mathrm{eq})$ is defined as one third of the trace of the orthogonalized Uij tensor.

\begin{tabular}{|c|c|c|c|c|}
\hline & $\mathrm{x}$ & Y & z & $\mathrm{U}(\mathrm{eq})$ \\
\hline $\operatorname{Mo}(1)$ & $582(1)$ & $4709(1)$ & $5974(1)$ & $26(1)$ \\
\hline Mo (2) & $5669(1)$ & $279(1)$ & $9027(1)$ & $27(1)$ \\
\hline Si (1) & $-350(2)$ & $5212(2)$ & $8085(1)$ & $38(1)$ \\
\hline $\mathrm{Si}(2)$ & $5205(2)$ & $-232(2)$ & $6902(1)$ & $44(1)$ \\
\hline$O(16)$ & $164(7)$ & $2357(4)$ & $7304(3)$ & $57(1)$ \\
\hline $\mathrm{O}(17)$ & $2833(5)$ & $3042(4)$ & $5192(3)$ & $47(1)$ \\
\hline $\mathrm{O}(18)$ & $-3474(5)$ & $4190(4)$ & $5953(3)$ & $43(1)$ \\
\hline$O(26)$ & $7566(6)$ & $1956(4)$ & $9819(3)$ & $47(1)$ \\
\hline $\mathrm{O}(27)$ & $1530(5)$ & $797(4)$ & $9018(3)$ & $43(1)$ \\
\hline$O(28)$ & $5117(7)$ & $2614(4)$ & $7672(3)$ & $59(1)$ \\
\hline$C(1)$ & $-2767(8)$ & $4958(7)$ & $7923(4)$ & $51(2)$ \\
\hline$C(2)$ & $-3938(9)$ & $4859(7)$ & $8690(5)$ & $55(2)$ \\
\hline$C(3)$ & $-5116(10)$ & $5586(8)$ & $8789(6)$ & $71(2)$ \\
\hline$C(4)$ & $761(10)$ & $3920(6)$ & $8797(4)$ & $54(2)$ \\
\hline$C(5)$ & $-185(10)$ & $6441(6)$ & $8517(5)$ & $59(2)$ \\
\hline$C(6)$ & $2713(9)$ & $20(7)$ & $7014(5)$ & $63(2)$ \\
\hline$C(7)$ & $1687(11)$ & $75(8)$ & $6266(6)$ & $75(2)$ \\
\hline$C(8)$ & $724(14)$ & $-693(10)$ & $6176(8)$ & $101(3)$ \\
\hline$C(9)$ & $6304(10)$ & $1042(7)$ & $6194(5)$ & $61(2)$ \\
\hline$C(10)$ & $5619(11)$ & $-1475(7)$ & $6467(5)$ & $68(2)$ \\
\hline$C(11)$ & $1484(8)$ & $6635(5)$ & $5645(4)$ & $37(1)$ \\
\hline $\mathrm{C}(12)$ & $167(7)$ & $6437(5)$ & $6291(4)$ & $34(1)$ \\
\hline$C(13)$ & $792(7)$ & $5601(5)$ & $7038(3)$ & $32(1)$ \\
\hline$C(14)$ & $2584(7)$ & $5283(5)$ & $6805(4)$ & $36(1)$ \\
\hline$C(15)$ & $3003(7)$ & $5915(5)$ & $5973(4)$ & $39(1)$ \\
\hline C (16) & $362(7)$ & $3221(5)$ & $6823(4)$ & $36(1)$ \\
\hline$C(17)$ & $1946(7)$ & $3667(5)$ & $5447(3)$ & $35(1)$ \\
\hline $\mathrm{C}(18)$ & $-1992(7)$ & $4391(5)$ & $5914(3)$ & $30(1)$ \\
\hline C (21) & $6219(7)$ & $-612(5)$ & $7983(4)$ & $35(1)$ \\
\hline $\mathrm{C}(22)$ & $7881(7)$ & $-307(5)$ & $8222(4)$ & $39(1)$ \\
\hline$C(23)$ & $8232(7)$ & $-932(5)$ & $9070(4)$ & $38(1)$ \\
\hline C (24) & $6772(8)$ & $-1650(5)$ & $9362(4)$ & $39(1)$ \\
\hline
\end{tabular}




$\begin{array}{llrll}\mathrm{C}(25) & 5535(8) & -1462(5) & 8707(4) & 38(1) \\ \mathrm{C}(26) & 6802(7) & 1331(5) & 9567(4) & 35(1) \\ \mathrm{C}(27) & 3048(7) & 585(5) & 9066(3) & 31(1) \\ \mathrm{C}(28) & 5348(8) & 1750(5) & 8177(4) & 39(1)\end{array}$

Table 3. Bond lengths [A] and angles [deg] for 9.

\begin{tabular}{|c|c|}
\hline $\operatorname{Mo}(1)-C(16)$ & $1.986(6)$ \\
\hline $\mathrm{Mo}(1)-\mathrm{C}(17)$ & $2.000(6)$ \\
\hline $\operatorname{Mo}(1)-\mathrm{C}(18)$ & $1.999(5)$ \\
\hline $\operatorname{Mo}(1)-C(14)$ & $2.331(6)$ \\
\hline $\operatorname{Mo}(1)-\mathrm{C}(13)$ & $2.355(5)$ \\
\hline $\operatorname{Mo}(1)-\mathrm{C}(12)$ & $2.358(5)$ \\
\hline $\mathrm{Mo}(1)-\mathrm{C}(15)$ & $2.399(6)$ \\
\hline $\operatorname{Mo}(1)-C(11)$ & $2.413(6)$ \\
\hline $\operatorname{Mo}(2)-C(28)$ & $1.974(6)$ \\
\hline $\operatorname{Mo}(2)-C(27)$ & $1.994(5)$ \\
\hline $\operatorname{Mo}(2)-C(26)$ & $2.008(6)$ \\
\hline $\operatorname{Mo}(2)-C(21)$ & $2.335(6)$ \\
\hline $\operatorname{Mo}(2)-C(22)$ & $2.336(6)$ \\
\hline $\operatorname{Mo}(2)-C(25)$ & $2.373(6)$ \\
\hline $\operatorname{Mo}(2)-C(23)$ & $2.384(5)$ \\
\hline $\operatorname{Mo}(2)-C(24)$ & $2.412(5)$ \\
\hline $\mathrm{Si}(1)-\mathrm{C}(13)$ & $1.871(6)$ \\
\hline $\mathrm{Si}(1)-\mathrm{C}(4)$ & $1.876(7)$ \\
\hline $\mathrm{Si}(1)-\mathrm{C}(5)$ & $1.872(7)$ \\
\hline $\mathrm{Si}(1)-\mathrm{C}(1)$ & $1.893(6)$ \\
\hline $\mathrm{Si}(2)-\mathrm{C}(9)$ & $1.885(8)$ \\
\hline $\mathrm{Si}(2)-\mathrm{C}(10)$ & $1.895(7)$ \\
\hline $\mathrm{Si}(2)-\mathrm{C}(6)$ & $1.900(7)$ \\
\hline $\mathrm{Si}(2)-\mathrm{C}(21)$ & $1.899(6)$ \\
\hline $\mathrm{O}(16)-\mathrm{C}(16)$ & $1.152(7)$ \\
\hline $\mathrm{O}(17)-\mathrm{C}(17)$ & $1.167(7)$ \\
\hline $\mathrm{O}(18)-\mathrm{C}(18)$ & $1.146(6)$ \\
\hline$O(26)-C(26)$ & $1.157(7)$ \\
\hline $\mathrm{O}(27)-\mathrm{C}(27)$ & $1.160(6)$ \\
\hline $\mathrm{O}(28)-\mathrm{C}(28)$ & $1.166(7)$ \\
\hline$C(1)-C(2)$ & $1.514(9)$ \\
\hline$C(2)-C(3)$ & $1.280(10)$ \\
\hline$C(6)-C(7)$ & $1.465(10)$ \\
\hline$C(7)-C(8)$ & $1.263(13)$ \\
\hline$C(11)-C(12)$ & $1.421(8)$ \\
\hline$C(11)-C(15)$ & $1.438(8)$ \\
\hline$C(12)-C(13)$ & $1.447(8)$ \\
\hline$C(13)-C(14)$ & $1.463(7)$ \\
\hline$C(14)-C(15)$ & $1.418(9)$ \\
\hline$C(21)-C(22)$ & $1.417(9)$ \\
\hline$C(21)-C(25)$ & $1.455(9)$ \\
\hline$C(22)-C(23)$ & $1.429(9)$ \\
\hline$C(23)-C(24)$ & $1.427(9)$ \\
\hline$C(24)-C(25)$ & $1.411(8)$ \\
\hline $\mathrm{C}(16)-\mathrm{Mo}(1)-\mathrm{C}(17)$ & $77.2(2)$ \\
\hline $\mathrm{C}(16)-\mathrm{Mo}(1)-\mathrm{C}(18)$ & $77.2(2)$ \\
\hline $\mathrm{C}(17)-\mathrm{Mo}(1)-\mathrm{C}(18)$ & $106.4(2)$ \\
\hline
\end{tabular}




\begin{tabular}{|c|c|}
\hline $\mathrm{C}(16)-\mathrm{Mo}(1)-\mathrm{C}(14)$ & $91.4(2)$ \\
\hline $\mathrm{C}(17)-\mathrm{Mo}(1)-\mathrm{C}(14)$ & $106.7(2)$ \\
\hline $\mathrm{C}(18)-\mathrm{Mo}(1)-\mathrm{C}(14)$ & $141.5(2)$ \\
\hline $\mathrm{C}(16)-\mathrm{Mo}(1)-\mathrm{C}(13)$ & $90.8(2)$ \\
\hline $\mathrm{C}(17)-\mathrm{Mo}(1)-\mathrm{C}(13)$ & $141.6(2)$ \\
\hline $\mathrm{C}(18)-\mathrm{Mo}(1)-\mathrm{C}(13)$ & $106.2(2)$ \\
\hline $\mathrm{C}(14)-\mathrm{Mo}(1)-\mathrm{C}(13)$ & $36.38(19)$ \\
\hline $\mathrm{C}(16)-\mathrm{Mo}(1)-\mathrm{C}(12)$ & $123.2(2)$ \\
\hline $\mathrm{C}(17)-\mathrm{Mo}(1)-\mathrm{C}(12)$ & $152.2(2)$ \\
\hline $\mathrm{C}(18)-\mathrm{Mo}(1)-\mathrm{C}(12)$ & $97.3(2)$ \\
\hline $\mathrm{C}(14)-\mathrm{Mo}(1)-\mathrm{C}(12)$ & $58.46(19)$ \\
\hline $\mathrm{C}(13)-\mathrm{Mo}(1)-\mathrm{C}(12)$ & $35.75(19)$ \\
\hline $\mathrm{C}(16)-\mathrm{Mo}(1)-\mathrm{C}(15)$ & $122.1(2)$ \\
\hline $\mathrm{C}(17)-\mathrm{Mo}(1)-\mathrm{C}(15)$ & $96.1(2)$ \\
\hline $\mathrm{C}(18)-\mathrm{Mo}(1)-\mathrm{C}(15)$ & $153.6(2)$ \\
\hline $\mathrm{C}(14)-\mathrm{Mo}(1)-\mathrm{C}(15)$ & $34.8(2)$ \\
\hline $\mathrm{C}(13)-\mathrm{Mo}(1)-\mathrm{C}(15)$ & $59.6(2)$ \\
\hline $\mathrm{C}(12)-\mathrm{Mo}(1)-\mathrm{C}(15)$ & $57.74(19)$ \\
\hline $\mathrm{C}(16)-\mathrm{Mo}(1)-\mathrm{C}(11)$ & $148.2(2)$ \\
\hline $\mathrm{C}(17)-\mathrm{Mo}(1)-\mathrm{C}(11)$ & $118.1(2)$ \\
\hline $\mathrm{C}(18)-\mathrm{Mo}(1)-\mathrm{C}(11)$ & $119.4(2)$ \\
\hline $\mathrm{C}(14)-\mathrm{Mo}(1)-\mathrm{C}(11)$ & $58.2(2)$ \\
\hline $\mathrm{C}(13)-\mathrm{Mo}(1)-\mathrm{C}(11)$ & $59.5(2)$ \\
\hline $\mathrm{C}(12)-\mathrm{Mo}(1)-\mathrm{C}(11)$ & $34.6(2)$ \\
\hline $\mathrm{C}(15)-\mathrm{Mo}(1)-\mathrm{C}(11)$ & $34.8(2)$ \\
\hline $\mathrm{C}(28)-\mathrm{Mo}(2)-\mathrm{C}(27)$ & $77.1(2)$ \\
\hline $\mathrm{C}(28)-\mathrm{Mo}(2)-\mathrm{C}(26)$ & $77.6(2)$ \\
\hline $\mathrm{C}(27)-\mathrm{Mo}(2)-\mathrm{C}(26)$ & $106.4(2)$ \\
\hline $\mathrm{C}(28)-\mathrm{Mo}(2)-\mathrm{C}(21)$ & $90.8(2)$ \\
\hline $\mathrm{C}(27)-\mathrm{Mo}(2)-\mathrm{C}(21)$ & $107.3(2)$ \\
\hline $\mathrm{C}(26)-\mathrm{Mo}(2)-\mathrm{C}(21)$ & $140.8(2)$ \\
\hline $\mathrm{C}(28)-\mathrm{Mo}(2)-\mathrm{C}(22)$ & $91.8(2)$ \\
\hline $\mathrm{C}(27)-\mathrm{Mo}(2)-\mathrm{C}(22)$ & $141.6(2)$ \\
\hline $\mathrm{C}(26)-\mathrm{Mo}(2)-\mathrm{C}(22)$ & $106.9(2)$ \\
\hline $\mathrm{C}(21)-\mathrm{Mo}(2)-\mathrm{C}(22)$ & $35.3(2)$ \\
\hline $\mathrm{C}(28)-\mathrm{Mo}(2)-\mathrm{C}(25)$ & $122.8(2)$ \\
\hline $\mathrm{C}(27)-\mathrm{Mo}(2)-\mathrm{C}(25)$ & $96.9(2)$ \\
\hline$C(26)-\operatorname{Mo}(2)-C(25)$ & $152.5(2)$ \\
\hline$C(21)-\operatorname{Mo}(2)-C(25)$ & $36.0(2)$ \\
\hline $\mathrm{C}(22)-\mathrm{Mo}(2)-\mathrm{C}(25)$ & $58.4(2)$ \\
\hline $\mathrm{C}(28)-\mathrm{Mo}(2)-\mathrm{C}(23)$ & $123.0(2)$ \\
\hline $\mathrm{C}(27)-\mathrm{Mo}(2)-\mathrm{C}(23)$ & $153.0(2)$ \\
\hline $\mathrm{C}(26)-\mathrm{Mo}(2)-\mathrm{C}(23)$ & $96.3(2)$ \\
\hline $\mathrm{C}(21)-\mathrm{Mo}(2)-\mathrm{C}(23)$ & $59.0(2)$ \\
\hline $\mathrm{C}(22)-\operatorname{Mo}(2)-\mathrm{C}(23)$ & $35.2(2)$ \\
\hline $\mathrm{C}(25)-\mathrm{Mo}(2)-\mathrm{C}(23)$ & $57.8(2)$ \\
\hline $\mathrm{C}(28)-\mathrm{Mo}(2)-\mathrm{C}(24)$ & $148.0(2)$ \\
\hline $\mathrm{C}(27)-\mathrm{Mo}(2)-\mathrm{C}(24)$ & $118.8(2)$ \\
\hline $\mathrm{C}(26)-\mathrm{Mo}(2)-\mathrm{C}(24)$ & $118.8(2)$ \\
\hline $\mathrm{C}(21)-\mathrm{Mo}(2)-\mathrm{C}(24)$ & $58.7(2)$ \\
\hline $\mathrm{C}(22)-\mathrm{Mo}(2)-\mathrm{C}(24)$ & $58.0(2)$ \\
\hline $\mathrm{C}(25)-\mathrm{Mo}(2)-\mathrm{C}(24)$ & $34.3(2)$ \\
\hline $\mathrm{C}(23)-\mathrm{Mo}(2)-\mathrm{C}(24)$ & $34.6(2)$ \\
\hline$C(13)-S i(1)-C(4)$ & $110.2(3)$ \\
\hline$C(13)-S i(1)-C(5)$ & $106.9(3)$ \\
\hline$C(4)-S i(1)-C(5)$ & $110.6(3)$ \\
\hline$C(13)-S i(1)-C(1)$ & $108.0(3)$ \\
\hline$C(4)-S i(1)-C(1)$ & $110.9(4)$ \\
\hline$C(5)-S i(1)-C(1)$ & $110.2(3)$ \\
\hline$C(9)-S i(2)-C(10)$ & $109.3(4)$ \\
\hline$C(9)-S i(2)-C(6)$ & $112.1(4)$ \\
\hline
\end{tabular}




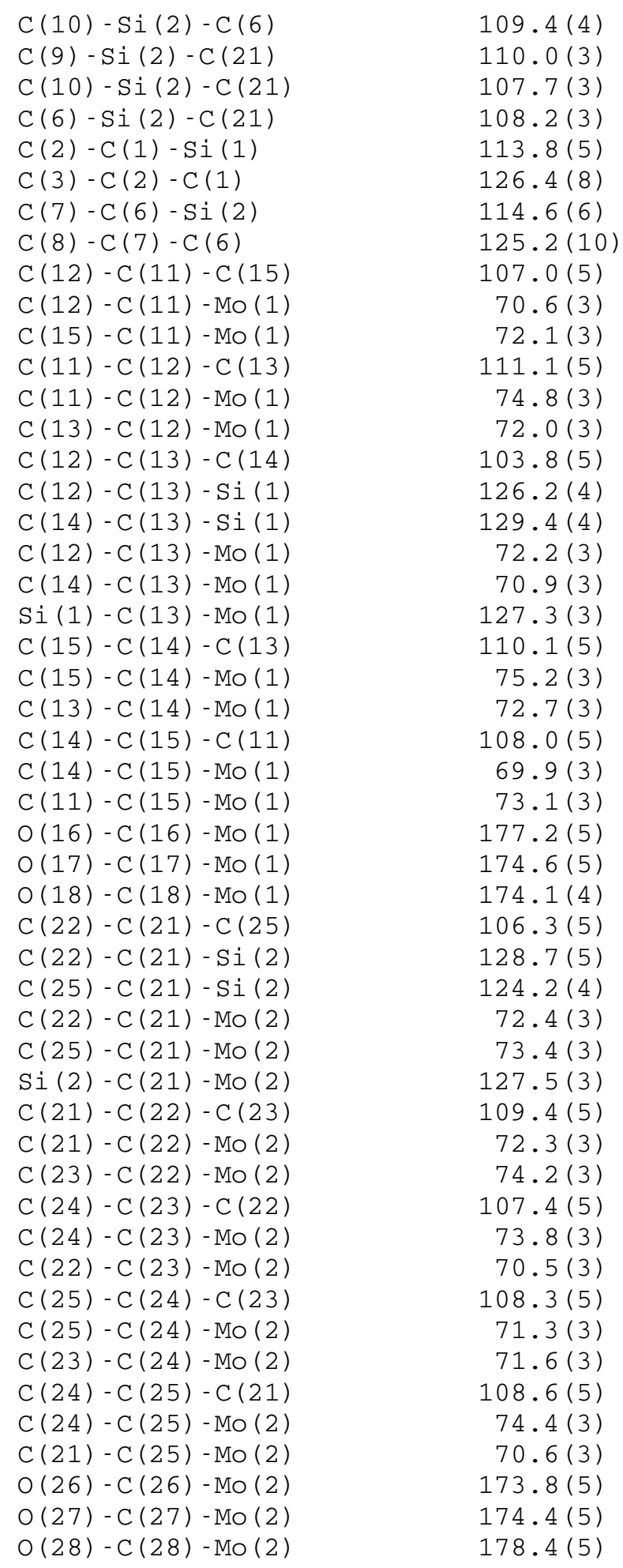

$109.4(4)$

$110.0(3)$

$107.7(3)$

$108.2(3)$

$113.8(5)$

$126.4(8)$

$114.6(6)$

$125.2(10)$

$107.0(5)$

$70.6(3)$

$72.1(3)$

$111.1(5)$

$74.8(3)$

$72.0(3)$

$103.8(5)$

$126.2(4)$

$129.4(4)$

$72.2(3)$

$70.9(3)$

$127.3(3)$

$110.1(5)$

$75.2(3)$

$72.7(3)$

$108.0(5)$

$69.9(3)$

$73.1(3)$

177.2 (5)

$174.6(5)$

$174.1(4)$

$106.3(5)$

128.7 (5)

$124.2(4)$

$72.4(3)$

$73.4(3)$

$127.5(3)$

$109.4(5)$

$72.3(3)$

$74.2(3)$

$107.4(5)$

$73.8(3)$

$70.5(3)$

$108.3(5)$

$71.3(3)$

$71.6(3)$

$108.6(5)$

$74.4(3)$

$70.6(3)$

$173.8(5)$

$174.4(5)$

$178.4(5)$

Symmetry transformations used to generate equivalent atoms: 
Table 4. Anisotropic displacement parameters $(A \wedge 2 \times 10 \wedge 3)$ for 9. The anisotropic displacement factor exponent takes the form: $-2 \mathrm{pi} \wedge 2[\mathrm{~h} \wedge 2 \mathrm{a} * \wedge 2 \mathrm{U} 11+\ldots+2 \mathrm{~h} \mathrm{k} a * b * \mathrm{U} 12]$

\begin{tabular}{|c|c|c|c|c|c|c|}
\hline & U11 & U2 2 & U33 & $\mathrm{U} 23$ & U13 & U12 \\
\hline Mo (1) & $34(1)$ & $22(1)$ & $19(1)$ & $-2(1)$ & $2(1)$ & $4(1)$ \\
\hline Mo (2) & $34(1)$ & $21(1)$ & $23(1)$ & $-1(1)$ & $1(1)$ & $0(1)$ \\
\hline $\mathrm{Si}(1)$ & $48(1)$ & $40(1)$ & $28(1)$ & $-12(1)$ & $5(1)$ & $-2(1)$ \\
\hline $\mathrm{Si}(2)$ & $58(1)$ & $44(1)$ & $31(1)$ & $-14(1)$ & $-7(1)$ & $12(1)$ \\
\hline$O(16)$ & $92(4)$ & $30(3)$ & $38(3)$ & $8(2)$ & $0(2)$ & $2(2)$ \\
\hline O (17) & $57(3)$ & $43(3)$ & $39(3)$ & $-10(2)$ & $7(2)$ & $18(2)$ \\
\hline O (18) & $46(3)$ & $47(3)$ & $31(2)$ & $-3(2)$ & $6(2)$ & $-5(2)$ \\
\hline$O(26)$ & $62(3)$ & $37(2)$ & $41(3)$ & $-8(2)$ & $-3(2)$ & $-18(2)$ \\
\hline$O(27)$ & $37(2)$ & $47(3)$ & $41(3)$ & $-8(2)$ & $-4(2)$ & $5(2)$ \\
\hline$O(28)$ & $90(3)$ & $33(3)$ & $43(3)$ & $9(2)$ & $0(2)$ & $-1(2)$ \\
\hline$C(1)$ & $53(4)$ & $67(5)$ & $42(4)$ & $-32(4)$ & $13(3)$ & $-10(3)$ \\
\hline$C(2)$ & $59(4)$ & $64(5)$ & $39(4)$ & $-11(4)$ & $10(3)$ & $-6(3)$ \\
\hline$C(3)$ & $61(4)$ & $89(6)$ & $71(6)$ & $-37(5)$ & $15(4)$ & $-2(4)$ \\
\hline$C(4)$ & $80(5)$ & $49(4)$ & $27(3)$ & $-3(3)$ & $6(3)$ & $-7(3)$ \\
\hline$C(5)$ & $73(4)$ & $58(5)$ & $52(5)$ & $-29(4)$ & $2(3)$ & $3(4)$ \\
\hline$C(6)$ & $74(5)$ & $71(5)$ & $50(5)$ & $-29(4)$ & $-19(4)$ & $18(4)$ \\
\hline$C(7)$ & $76(5)$ & $63(5)$ & $85(7)$ & $-22(5)$ & $-30(5)$ & $6(4)$ \\
\hline$C(8)$ & $121(8)$ & $83(7)$ & $106(9)$ & $-36(7)$ & $-24(6)$ & $-22(6)$ \\
\hline$C(9)$ & $87(5)$ & $51(4)$ & $37(4)$ & $-1(3)$ & $-4(3)$ & $7(4)$ \\
\hline$C(10)$ & $95(6)$ & $68(5)$ & $48(5)$ & $-31(4)$ & $-8(4)$ & $14(4)$ \\
\hline$C(11)$ & $54(3)$ & $27(3)$ & $27(3)$ & $-5(2)$ & $4(2)$ & $-5(2)$ \\
\hline$C(12)$ & $39(3)$ & $26(3)$ & $36(3)$ & $-8(3)$ & $-1(2)$ & $2(2)$ \\
\hline$C(13)$ & $40(3)$ & $29(3)$ & $25(3)$ & $-8(2)$ & $1(2)$ & $0(2)$ \\
\hline$C(14)$ & $39(3)$ & $34(3)$ & $31(3)$ & $-5(3)$ & $-1(2)$ & $0(2)$ \\
\hline$C(15)$ & $33(3)$ & $47(4)$ & $38(3)$ & $-14(3)$ & $9(2)$ & $-6(2)$ \\
\hline$C(16)$ & $48(3)$ & $32(3)$ & $24(3)$ & $-3(3)$ & $-1(2)$ & $6(2)$ \\
\hline$C(17)$ & $44(3)$ & $32(3)$ & $24(3)$ & $-2(2)$ & $-4(2)$ & $4(2)$ \\
\hline$C(18)$ & $27(3)$ & $36(3)$ & $22(3)$ & $0(2)$ & $2(2)$ & $5(2)$ \\
\hline$C(21)$ & $44(3)$ & $28(3)$ & $34(3)$ & $-9(3)$ & $2(2)$ & $5(2)$ \\
\hline $\mathrm{C}(22)$ & $43(3)$ & $36(3)$ & $36(3)$ & $-8(3)$ & $4(2)$ & $3(2)$ \\
\hline$C(23)$ & $35(3)$ & $42(3)$ & $36(3)$ & $-10(3)$ & $-4(2)$ & $10(2)$ \\
\hline$C(24)$ & $54(3)$ & $27(3)$ & $32(3)$ & $-5(3)$ & $0(2)$ & $9(2)$ \\
\hline$C(25)$ & $44(3)$ & $29(3)$ & $42(4)$ & $-12(3)$ & $2(2)$ & $3(2)$ \\
\hline$C(26)$ & $42(3)$ & $30(3)$ & $26(3)$ & $3(2)$ & $6(2)$ & $-4(2)$ \\
\hline$C(27)$ & $29(3)$ & $35(3)$ & $24(3)$ & $-2(2)$ & $-1(2)$ & $-5(2)$ \\
\hline$C(28)$ & $47(3)$ & $33(3)$ & $35(3)$ & $-6(3)$ & $4(2)$ & $0(2)$ \\
\hline
\end{tabular}

